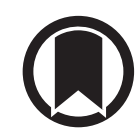

CrossMark

\title{
Low-dose computed tomography in elderly pneumonia patients: a need to individualise
}

To the Editor:

We read with interest the study by PRENDKI et al. [1], in which the authors proved the diagnostic role of low-dose computed tomography (LDCT) of the thorax in the evaluation of elderly patients with pneumonia. The results showed that addition of LDCT modified the probability of pneumonia in $45 \%$ of the patients. However, on comparing with the reference diagnosis, a high discordance rate of $32.2 \%$ was found in the LDCT-based diagnosis that needs to be justified [1]. Being an interventional study, the authors should mention the treatment outcomes of all the enrolled patients, particularly those with low probability after LDCT (treated with or without antibiotics). Comparing these outcomes among low/ intermediate/high diagnostic groups before and after LDCT might help to reveal a clear picture. Moreover, in view of clinician-labelled diagnosis being the primary outcome in the study, using multiple physicians for better diagnostic agreement might have increased the authenticity of the results.

LDCT has a specific place in the diagnostic algorithm for pneumonia [2] and may even be detrimental if used injudiciously. Consolidation in elderly patients encompasses a variety of other differential diagnoses, notably lung cancer, metastasis and diffuse parenchymal lung diseases, which are likely to be missed if we use LDCT without appropriate settings (like contrast, high-resolution cuts, etc.). This is especially true because clinical features in the elderly are neither sensitive nor specific in differentiating infective from non-infective aetiologies. With narrow differentials, prominent symptoms and concern for radiation exposure, LDCT may find a better place in the evaluation of pneumonia in young patients, but this needs future research.

Computed tomography is an important but not a gold standard diagnostic tool in pneumonia [3]. Considering the wide array of possible aetiologies in elderly patients, the decision to use LDCT, conventional computed tomography or no computed tomography should be individualised based on the clinical scenario, ongoing treatment response, availability of computed tomography and its cost-effectiveness.

@ERSpublications

Low-dose computed tomography is an important diagnostic tool in elderly patients with pneumonia; however, its use should be individualised based on patient- and disease-related factors http://ow.ly/sqZs30kuLEX

Cite this article as: Aggarwal D, Saini V, Gour H. Low-dose computed tomography in elderly pneumonia patients: a need to individualise. Eur Respir J 2018; 52: 1800815 [https://doi.org/10.1183/13993003.00815-2018].

Deepak Aggarwal, Varinder Saini and Hitesh Gour

Dept of Pulmonary Medicine, Government Medical College and Hospital, Sector 32, Chandigarh, India.

Correspondence: Deepak Aggarwal, Dept of Pulmonary Medicine, Block D, Level 5, Government Medical College and Hospital, Sector 32, Chandigarh, 160030, India. E-mail: drdeepak@hotmail.com

Received: April 302018 | Accepted after revision: June 052018

Conflict of interest: None declared.

\section{References}

1 Prendki V, Scheffler M, Huttner B, et al. Low-dose computed tomography for the diagnosis of pneumonia in elderly patients: a prospective, interventional cohort study. Eur Respir J 2018; 51: 1702375.

2 Claessens YE, Debray MP, Tubach F, et al. Early chest computed tomography scan to assist diagnosis and guide treatment decision for suspected community-acquired pneumonia. Am J Respir Crit Care Med 2015; 192: 974-982.

3 Waterer GW. The diagnosis of community-acquired pneumonia. Do we need to take a big step backward? Am J Respir Crit Care Med 2015; 192: 912-913. 